\title{
Supporting information for \\ "Theoretical study on the second hyperpolarizabilities of phenalenyl radical systems involving acetylene and vinylene linkers: diradical character and spin multiplicity dependences"
}

Suguru Ohta*, , Masayoshi Nakano*,†, Takashi Kubo ${ }^{\ddagger}, K_{\text {Kenji Kamada }}^{\S}$, Koji Ohta ${ }^{\S}$, Ryohei Kishi $^{\dagger}$, Nozomi Nakagawa ${ }^{\dagger}$, Benoît Champagne", Edith Botek ${ }^{\prime \prime}$, Akihito Takebe ${ }^{\dagger}$, Shin-ya Umezaki $^{\dagger}$, Masahito Nate ${ }^{\dagger}$, Hideaki Takahashi ${ }^{\dagger}$, Shin-ichi Furukawa ${ }^{\dagger}$, Yasushi Morita ${ }^{\ddagger}$, Kazuhiro Nakasuji ${ }^{\ddagger}$, Kizashi Yamaguchi ${ }^{\ddagger}$

${ }^{\dagger}$ Department of Materials Engineering Science, Graduate School of Engineering Science, Osaka University, Toyonaka, Osaka 560-8531, Japan.

${ }^{\ddagger}$ Department of Chemistry, Graduate School of Science, Osaka University, Toyonaka, Osaka 560-0043, Japan.

${ }^{\S}$ Photonics Research Institute, National Institute of Advanced Industrial Science and Technology (AIST), Ikeda, Osaka 563-8577, Japan

" Laboratoire de Chimie Théorique Appliquée, Facultés Universitaires Notre-Dame de la Paix, rue de Bruxelles, 61, 5000 Namur, Belgium

E-mail: s_ohta@cheng.es.osaka-u.ac.jp (S.O.), mnaka@cheng.es.osaka-u.ac.jp (M.N.) 


\section{Contents}

1. Diradical characters of dipyrenyl molecules $\mathbf{3}$ and $\mathbf{4}$

2. Table 1S: Cartesian coordinates of 1(singlet) calculated at the UB3LYP/6-31G** level of approximation

3. Table 2S: Cartesian coordinates of 2(singlet) calculated at the UB3LYP/6-31G** level of approximation

4. Table 3S: Cartesian coordinates of $\mathbf{3}$ calculated at the RB3LYP/6-31G** level of approximation

5. Table 4S: Cartesian coordinates of 4 calculated at the RB3LYP/6-31G** level of approximation

6. Table 5S: Cartesian coordinate of 5(singlet) calculated at the UB3LYP/6-31G** level of approximation

7. Table 6S: Cartesian coordinate of 6(singlet) calculated at the UB3LYP/6-31G** level of approximation

8. Table 7S: Cartesian coordinate of 1(triplet) calculated at the UB3LYP/6-31G** level of approximation

9. Table 8S: Cartesian coordinate of 2(triplet) calculated at the UB3LYP/6-31G** level of approximation

10. Table 9S: Cartesian coordinate of 5(triplet) calculated at the UB3LYP/6-31G** level of approximation

11. Table 10S: Cartesian coordinate of 6(triplet) calculated at the UB3LYP/6-31G** level of approximation

12. Table 11S: Spin expectation values $\left\langle S^{2}>\right.$ of $\mathbf{1}$ (singlet), 2(singlet), 5(singlet), $\mathbf{6}$ (singlet), 1(triplet), 2(triplet), 5(triplet), 6(triplet) evaluated at the UBHandHLYP/6-31G* level of approximation 
Diradical characters of dipyrenyl molecules 3 and $\mathbf{4}$

The diradical character $(y)$ of $\mathbf{3}$ (see Table 1) calculated from the $\mathrm{UNO} / 6-31 \mathrm{G}^{* *}$ is 0.1915 , which indicates the open-shell, but close to closed-shell, feature of this molecule. Therefore, the previous result of this molecule $(y=0.0)$ by the same method reported in our paper [BPRY1 in Chem. Phys. Lett. 2006, 420, 432-437] is found to be incorrect because of being obtained from the unstable spin-restricted (closed-shell) solution at the $\mathrm{HF} / 6-31 \mathrm{G} * *$ level. On the other hand, at the BHandHLYP/6-31G* level, this molecule is found to have a stable spin-restricted (closed-shell) solution, so that the $\gamma$ value of this molecule is the same as the previous result at the RBHandHLYP/6-31G* level. The similar feature is observed for 4 ( $y=0.2297$ and $\gamma=324$ a.u.). These results suggest that these molecules with small diradical character less than about $y \sim 0.2$ are actually regarded as closed-shell systems at the BHandHLYP/6-31G* level. Indeed, the RBHandHLYP method is known to provide reliable $\gamma$ value in that small diradical character or closed-shell regime [ref. 21(a)]. 
Table 1S: Cartesian coordinates of $\mathbf{1}$ (singlet) calculated at the UB3LYP/6-31G** level of approximation

\begin{tabular}{|c|c|c|c|}
\hline Atom type & $\mathrm{X}$ & Y & $\mathrm{Z}$ \\
\hline $\mathrm{C}$ & 0.618475 & 0.000000 & 0.000000 \\
\hline $\mathrm{C}$ & -0.618475 & 0.000000 & 0.000000 \\
\hline $\mathrm{C}$ & 1.999952 & 0.006444 & 0.000000 \\
\hline $\mathrm{C}$ & -1.999952 & -0.006444 & 0.000000 \\
\hline $\mathrm{C}$ & 2.714191 & -1.233908 & 0.000000 \\
\hline $\mathrm{C}$ & -2.714191 & 1.233908 & 0.000000 \\
\hline $\mathrm{C}$ & 4.079652 & -1.276285 & 0.000000 \\
\hline $\mathrm{C}$ & -4.079652 & 1.276285 & 0.000000 \\
\hline $\mathrm{C}$ & 4.867271 & -0.075441 & 0.000000 \\
\hline $\mathrm{C}$ & -4.867271 & 0.075441 & 0.000000 \\
\hline $\mathrm{C}$ & 4.190528 & 1.185762 & 0.000000 \\
\hline $\mathrm{C}$ & -4.190528 & -1.185762 & 0.000000 \\
\hline $\mathrm{C}$ & 2.761259 & 1.249569 & 0.000000 \\
\hline $\mathrm{C}$ & -2.761259 & -1.249569 & 0.000000 \\
\hline $\mathrm{C}$ & 2.141100 & 2.507042 & 0.000000 \\
\hline $\mathrm{C}$ & -2.141100 & -2.507042 & 0.000000 \\
\hline $\mathrm{C}$ & 2.895621 & 3.685811 & 0.000000 \\
\hline $\mathrm{C}$ & -2.895621 & -3.685811 & 0.000000 \\
\hline $\mathrm{C}$ & 4.279308 & 3.638946 & 0.000000 \\
\hline $\mathrm{C}$ & -4.279308 & -3.638946 & 0.000000 \\
\hline $\mathrm{C}$ & 4.956884 & 2.394221 & 0.000000 \\
\hline $\mathrm{C}$ & -4.956884 & -2.394221 & 0.000000 \\
\hline $\mathrm{C}$ & 6.375620 & 2.310592 & 0.000000 \\
\hline $\mathrm{C}$ & -6.375620 & -2.310592 & 0.000000 \\
\hline $\mathrm{C}$ & 7.012969 & 1.083729 & 0.000000 \\
\hline $\mathrm{C}$ & -7.012969 & -1.083729 & 0.000000 \\
\hline
\end{tabular}




\begin{tabular}{lrrr}
$\mathrm{C}$ & 6.267163 & -0.104391 & 0.000000 \\
$\mathrm{C}$ & -6.267163 & 0.104391 & 0.000000 \\
$\mathrm{H}$ & 2.138123 & -2.153519 & 0.000000 \\
$\mathrm{H}$ & -2.138123 & 2.153519 & 0.000000 \\
$\mathrm{H}$ & 4.594555 & -2.232974 & 0.000000 \\
$\mathrm{H}$ & -4.594555 & 2.232974 & 0.000000 \\
$\mathrm{H}$ & 6.775818 & -1.064529 & 0.000000 \\
$\mathrm{H}$ & -6.775818 & 1.064529 & 0.000000 \\
$\mathrm{H}$ & 8.097914 & 1.035929 & 0.000000 \\
$\mathrm{H}$ & -8.097914 & -1.035929 & 0.000000 \\
$\mathrm{H}$ & 6.953196 & 3.230802 & 0.000000 \\
$\mathrm{H}$ & -6.953196 & -3.230802 & 0.000000 \\
$\mathrm{H}$ & 4.862468 & 4.555652 & 0.000000 \\
$\mathrm{H}$ & -4.862468 & -4.555652 & 0.000000 \\
$\mathrm{H}$ & 2.385880 & 4.644812 & 0.000000 \\
$\mathrm{H}$ & -2.385880 & -4.644812 & 0.000000 \\
$\mathrm{H}$ & 1.057329 & 2.556238 & 0.000000 \\
$\mathrm{H}$ & -1.057329 & -2.556238 & 0.000000 \\
\hline & & & \\
\hline & & & \\
\hline & & &
\end{tabular}


Table 2S: Cartesian coordinates of 2(singlet) calculated at the UB3LYP/6-31G** level of approximation

\begin{tabular}{|c|c|c|c|}
\hline Atom type & $X$ & $\mathrm{Y}$ & $\mathrm{Z}$ \\
\hline $\mathrm{C}$ & 0.611665 & -0.353145 & 0.000000 \\
\hline $\mathrm{C}$ & -0.611665 & 0.353145 & 0.000000 \\
\hline $\mathrm{C}$ & 1.893450 & 0.190054 & 0.000000 \\
\hline $\mathrm{C}$ & -1.893450 & -0.190054 & 0.000000 \\
\hline $\mathrm{C}$ & 4.397477 & -0.050601 & 0.000000 \\
\hline $\mathrm{C}$ & -4.397477 & 0.050601 & 0.000000 \\
\hline $\mathrm{C}$ & 3.098836 & -0.663943 & 0.000000 \\
\hline $\mathrm{C}$ & -3.098836 & 0.663943 & 0.000000 \\
\hline $\mathrm{C}$ & 5.579644 & -0.858287 & 0.000000 \\
\hline $\mathrm{C}$ & -5.579644 & 0.858287 & 0.000000 \\
\hline $\mathrm{C}$ & 4.538739 & 1.373614 & 0.000000 \\
\hline $\mathrm{C}$ & -4.538739 & -1.373614 & 0.000000 \\
\hline $\mathrm{C}$ & 2.107425 & 1.618668 & 0.000000 \\
\hline $\mathrm{C}$ & -2.107425 & -1.618668 & 0.000000 \\
\hline $\mathrm{C}$ & 6.964405 & 1.146565 & 0.000000 \\
\hline $\mathrm{C}$ & -6.964405 & -1.146565 & 0.000000 \\
\hline $\mathrm{C}$ & 4.203802 & -2.851412 & 0.000000 \\
\hline $\mathrm{C}$ & -4.203802 & 2.851412 & 0.000000 \\
\hline $\mathrm{C}$ & 3.341912 & 2.177929 & 0.000000 \\
\hline $\mathrm{C}$ & -3.341912 & -2.177929 & 0.000000 \\
\hline $\mathrm{C}$ & 6.854885 & -0.226994 & 0.000000 \\
\hline $\mathrm{C}$ & -6.854885 & 0.226994 & 0.000000 \\
\hline $\mathrm{C}$ & 3.044552 & -2.061843 & 0.000000 \\
\hline $\mathrm{C}$ & -3.044552 & 2.061843 & 0.000000 \\
\hline $\mathrm{C}$ & 5.807681 & 1.947258 & 0.000000 \\
\hline $\mathrm{C}$ & -5.807681 & -1.947258 & 0.000000 \\
\hline
\end{tabular}




\begin{tabular}{|c|c|c|c|}
\hline $\mathrm{C}$ & 5.454284 & -2.268154 & 0.000000 \\
\hline $\mathrm{C}$ & -5.454284 & 2.268154 & 0.000000 \\
\hline $\mathrm{H}$ & 0.543226 & -1.433776 & 0.000000 \\
\hline $\mathrm{H}$ & -0.543226 & 1.433776 & 0.000000 \\
\hline $\mathrm{H}$ & 1.243399 & 2.272773 & 0.000000 \\
\hline $\mathrm{H}$ & -1.243399 & -2.272773 & 0.000000 \\
\hline $\mathrm{H}$ & 7.943603 & 1.616234 & 0.000000 \\
\hline $\mathrm{H}$ & -7.943603 & -1.616234 & 0.000000 \\
\hline $\mathrm{H}$ & 4.107065 & -3.933129 & 0.000000 \\
\hline $\mathrm{H}$ & -4.107065 & 3.933129 & 0.000000 \\
\hline $\mathrm{H}$ & 3.451230 & 3.259204 & 0.000000 \\
\hline $\mathrm{H}$ & -3.451230 & -3.259204 & 0.000000 \\
\hline $\mathrm{H}$ & 5.898390 & 3.030144 & 0.000000 \\
\hline $\mathrm{H}$ & -5.898390 & -3.030144 & 0.000000 \\
\hline $\mathrm{H}$ & 7.743984 & -0.851520 & 0.000000 \\
\hline $\mathrm{H}$ & -7.743984 & 0.851520 & 0.000000 \\
\hline $\mathrm{H}$ & 6.352417 & -2.879405 & 0.000000 \\
\hline $\mathrm{H}$ & -6.352417 & 2.879405 & 0.000000 \\
\hline $\mathrm{H}$ & 2.088171 & -2.571547 & 0.000000 \\
\hline $\mathrm{H}$ & -2.088171 & 2.571547 & 0.000000 \\
\hline
\end{tabular}


Table 3S: Cartesian coordinates of $\mathbf{3}$ calculated at the RB3LYP/6-31G** level of approximation

\begin{tabular}{cccl}
\hline Atom type & $\mathrm{X}$ & $\mathrm{Y}$ & $\mathrm{Z}$ \\
\hline $\mathrm{C}$ & 0.609115 & 0.000000 & 0.000000 \\
$\mathrm{C}$ & -0.609115 & 0.000000 & 0.000000 \\
$\mathrm{C}$ & 2.751452 & 1.281757 & 0.000000 \\
$\mathrm{C}$ & -2.751452 & -1.281757 & 0.000000 \\
$\mathrm{C}$ & 4.179003 & 1.257801 & 0.000000 \\
$\mathrm{C}$ & -4.179003 & -1.257801 & 0.000000 \\
$\mathrm{C}$ & 4.885260 & 0.018308 & 0.000000 \\
$\mathrm{C}$ & -4.885260 & -0.018308 & 0.000000 \\
$\mathrm{C}$ & 4.160911 & -1.212357 & 0.000000 \\
$\mathrm{C}$ & -4.160911 & 1.212357 & 0.000000 \\
$\mathrm{C}$ & 2.732346 & -1.171144 & 0.000000 \\
$\mathrm{C}$ & -2.732346 & 1.171144 & 0.000000 \\
$\mathrm{C}$ & 2.031944 & 0.013566 & 0.000000 \\
$\mathrm{C}$ & -2.031944 & -0.013566 & 0.000000 \\
$\mathrm{C}$ & 6.312714 & 0.001125 & 0.000000 \\
$\mathrm{C}$ & -6.312714 & -0.001125 & 0.000000 \\
$\mathrm{C}$ & 7.016784 & 1.253885 & 0.000000 \\
$\mathrm{C}$ & -7.016784 & -1.253885 & 0.000000 \\
$\mathrm{C}$ & 6.345409 & 2.437807 & 0.000000 \\
$\mathrm{C}$ & -6.345409 & -2.437807 & 0.000000 \\
$\mathrm{C}$ & 4.908769 & 2.487063 & 0.000000 \\
$\mathrm{C}$ & -4.908769 & -2.487063 & 0.000000 \\
$\mathrm{C}$ & 4.204174 & 3.700879 & 0.000000 \\
$\mathrm{C}$ & -4.204174 & -3.700879 & 0.000000 \\
$\mathrm{C}$ & 2.812330 & 3.712014 & 0.000000 \\
$\mathrm{C}$ & -2.812330 & -3.712014 & 0.000000
\end{tabular}




\begin{tabular}{|c|c|c|c|}
\hline $\mathrm{C}$ & 2.091926 & 2.518486 & 0.000000 \\
\hline $\mathrm{C}$ & -2.091926 & -2.518486 & 0.000000 \\
\hline $\mathrm{C}$ & 4.872706 & -2.424755 & 0.000000 \\
\hline $\mathrm{C}$ & -4.872706 & 2.424755 & 0.000000 \\
\hline $\mathrm{C}$ & 6.264991 & -2.432452 & 0.000000 \\
\hline $\mathrm{C}$ & -6.264991 & 2.432452 & 0.000000 \\
\hline $\mathrm{C}$ & 6.979297 & -1.235263 & 0.000000 \\
\hline $\mathrm{C}$ & -6.979297 & 1.235263 & 0.000000 \\
\hline $\mathrm{H}$ & 2.187073 & -2.110091 & 0.000000 \\
\hline $\mathrm{H}$ & -2.187073 & 2.110091 & 0.000000 \\
\hline $\mathrm{H}$ & 6.890744 & 3.377860 & 0.000000 \\
\hline $\mathrm{H}$ & -6.890744 & -3.377860 & 0.000000 \\
\hline $\mathrm{H}$ & 4.759196 & 4.635118 & 0.000000 \\
\hline $\mathrm{H}$ & -4.759196 & -4.635118 & 0.000000 \\
\hline $\mathrm{H}$ & 8.103356 & 1.242729 & 0.000000 \\
\hline $\mathrm{H}$ & -8.103356 & -1.242729 & 0.000000 \\
\hline $\mathrm{H}$ & 4.315773 & -3.357738 & 0.000000 \\
\hline $\mathrm{H}$ & -4.315773 & 3.357738 & 0.000000 \\
\hline $\mathrm{H}$ & 6.798745 & -3.378163 & 0.000000 \\
\hline $\mathrm{H}$ & -6.798745 & 3.378163 & 0.000000 \\
\hline $\mathrm{H}$ & 8.065826 & -1.249332 & 0.000000 \\
\hline $\mathrm{H}$ & -8.065826 & 1.249332 & 0.000000 \\
\hline $\mathrm{H}$ & 2.280173 & 4.658700 & 0.000000 \\
\hline $\mathrm{H}$ & -2.280173 & -4.658700 & 0.000000 \\
\hline $\mathrm{H}$ & 1.007459 & 2.534417 & 0.000000 \\
\hline $\mathrm{H}$ & -1.007459 & -2.534417 & 0.000000 \\
\hline
\end{tabular}


Table 4S: Cartesian coordinates of 4 calculated at the RB3LYP/6-31G** level of approximation

\begin{tabular}{cccl}
\hline Atom type & $\mathrm{X}$ & $\mathrm{Y}$ & $\mathrm{Z}$ \\
\hline $\mathrm{C}$ & 0.585151 & -0.337837 & 0.000000 \\
$\mathrm{C}$ & -0.585151 & 0.337837 & 0.000000 \\
$\mathrm{C}$ & 4.586001 & 1.350411 & 0.000000 \\
$\mathrm{C}$ & -4.586001 & -1.350411 & 0.000000 \\
$\mathrm{C}$ & 4.416664 & -0.065728 & 0.000000 \\
$\mathrm{C}$ & -4.416664 & 0.065728 & 0.000000 \\
$\mathrm{C}$ & 3.437156 & 2.195237 & 0.000000 \\
$\mathrm{C}$ & -3.437156 & -2.195237 & 0.000000 \\
$\mathrm{C}$ & 5.577275 & -0.903096 & 0.000000 \\
$\mathrm{C}$ & -5.577275 & 0.903096 & 0.000000 \\
$\mathrm{C}$ & 5.890810 & 1.930887 & 0.000000 \\
$\mathrm{C}$ & -5.890810 & -1.930887 & 0.000000 \\
$\mathrm{C}$ & 3.105672 & -0.647869 & 0.000000 \\
$\mathrm{C}$ & -3.105672 & 0.647869 & 0.000000 \\
$\mathrm{C}$ & 1.936619 & 0.234896 & 0.000000 \\
$\mathrm{C}$ & -1.936619 & -0.234896 & 0.000000 \\
$\mathrm{C}$ & 7.031542 & 1.058456 & 0.000000 \\
$\mathrm{C}$ & -7.031542 & -1.058456 & 0.000000 \\
$\mathrm{C}$ & 2.143630 & 1.594945 & 0.000000 \\
$\mathrm{C}$ & -2.143630 & -1.594945 & 0.000000 \\
$\mathrm{C}$ & 6.878902 & -0.292897 & 0.000000 \\
$\mathrm{C}$ & -6.878902 & 0.292897 & 0.000000 \\
$\mathrm{C}$ & 3.014913 & -2.051131 & 0.000000 \\
$\mathrm{C}$ & -3.014913 & 2.051131 & 0.000000 \\
$\mathrm{C}$ & 6.016480 & 3.329409 & 0.000000 \\
$\mathrm{C}$ & -6.016480 & -3.329409 & 0.000000 \\
& & & \\
\hline & & & \\
\hline & & &
\end{tabular}




\begin{tabular}{|c|c|c|c|}
\hline $\mathrm{C}$ & 5.421816 & -2.296439 & 0.000000 \\
\hline $\mathrm{C}$ & -5.421816 & 2.296439 & 0.000000 \\
\hline $\mathrm{C}$ & 3.611808 & 3.591103 & 0.000000 \\
\hline $\mathrm{C}$ & -3.611808 & -3.591103 & 0.000000 \\
\hline $\mathrm{C}$ & 4.150962 & -2.858478 & 0.000000 \\
\hline $\mathrm{C}$ & -4.150962 & 2.858478 & 0.000000 \\
\hline $\mathrm{C}$ & 4.887559 & 4.147674 & 0.000000 \\
\hline $\mathrm{C}$ & -4.887559 & -4.147674 & 0.000000 \\
\hline $\mathrm{H}$ & 0.536522 & -1.419626 & 0.000000 \\
\hline $\mathrm{H}$ & -0.536522 & 1.419626 & 0.000000 \\
\hline $\mathrm{H}$ & 1.292835 & 2.269237 & 0.000000 \\
\hline $\mathrm{H}$ & -1.292835 & -2.269237 & 0.000000 \\
\hline $\mathrm{H}$ & 2.735258 & 4.233322 & 0.000000 \\
\hline $\mathrm{H}$ & -2.735258 & -4.233322 & 0.000000 \\
\hline $\mathrm{H}$ & 5.004928 & 5.227330 & 0.000000 \\
\hline $\mathrm{H}$ & -5.004928 & -5.227330 & 0.000000 \\
\hline $\mathrm{H}$ & 7.009641 & 3.770287 & 0.000000 \\
\hline $\mathrm{H}$ & -7.009641 & -3.770287 & 0.000000 \\
\hline $\mathrm{H}$ & 8.023486 & 1.502305 & 0.000000 \\
\hline $\mathrm{H}$ & -8.023486 & -1.502305 & 0.000000 \\
\hline $\mathrm{H}$ & 7.748567 & -0.944623 & 0.000000 \\
\hline $\mathrm{H}$ & -7.748567 & 0.944623 & 0.000000 \\
\hline $\mathrm{H}$ & 6.304441 & -2.930124 & 0.000000 \\
\hline $\mathrm{H}$ & -6.304441 & 2.930124 & 0.000000 \\
\hline $\mathrm{H}$ & 4.036311 & -3.938425 & 0.000000 \\
\hline $\mathrm{H}$ & -4.036311 & 3.938425 & 0.000000 \\
\hline $\mathrm{H}$ & 2.048315 & -2.539017 & 0.000000 \\
\hline $\mathrm{H}$ & -2.048315 & 2.539017 & 0.000000 \\
\hline
\end{tabular}


Table 5S: Cartesian coordinate of 5(singlet) calculated at the UB3LYP/6-31G** level of approximation

\begin{tabular}{|c|c|c|c|}
\hline Atom type & $X$ & Y & $\mathrm{Z}$ \\
\hline $\mathrm{C}$ & 0.608163 & 0.000000 & 0.000000 \\
\hline $\mathrm{C}$ & -0.608163 & 0.000000 & 0.000000 \\
\hline $\mathrm{C}$ & 2.033837 & 0.000000 & 0.000000 \\
\hline $\mathrm{C}$ & -2.033837 & 0.000000 & 0.000000 \\
\hline $\mathrm{C}$ & 4.872756 & 0.000000 & 0.000000 \\
\hline $\mathrm{C}$ & -4.872756 & 0.000000 & 0.000000 \\
\hline $\mathrm{C}$ & 4.155314 & 1.238176 & 0.000000 \\
\hline $\mathrm{C}$ & -4.155314 & -1.238176 & 0.000000 \\
\hline $\mathrm{C}$ & 4.155314 & -1.238176 & 0.000000 \\
\hline $\mathrm{C}$ & -4.155314 & 1.238176 & 0.000000 \\
\hline $\mathrm{C}$ & 6.302577 & 0.000000 & 0.000000 \\
\hline $\mathrm{C}$ & -6.302577 & 0.000000 & 0.000000 \\
\hline $\mathrm{C}$ & 2.741724 & 1.214229 & 0.000000 \\
\hline $\mathrm{C}$ & -2.741724 & -1.214229 & 0.000000 \\
\hline $\mathrm{C}$ & 2.741724 & -1.214229 & 0.000000 \\
\hline $\mathrm{C}$ & -2.741724 & 1.214229 & 0.000000 \\
\hline $\mathrm{C}$ & 4.889134 & 2.453269 & 0.000000 \\
\hline $\mathrm{C}$ & -4.889134 & -2.453269 & 0.000000 \\
\hline $\mathrm{C}$ & 4.889134 & -2.453269 & 0.000000 \\
\hline $\mathrm{C}$ & -4.889134 & 2.453269 & 0.000000 \\
\hline $\mathrm{C}$ & 6.986047 & 1.243165 & 0.000000 \\
\hline $\mathrm{C}$ & -6.986047 & -1.243165 & 0.000000 \\
\hline $\mathrm{C}$ & 6.986047 & -1.243165 & 0.000000 \\
\hline $\mathrm{C}$ & -6.986047 & 1.243165 & 0.000000 \\
\hline $\mathrm{C}$ & 6.281080 & 2.444108 & 0.000000 \\
\hline $\mathrm{C}$ & -6.281080 & -2.444108 & 0.000000 \\
\hline
\end{tabular}




\begin{tabular}{lrrr}
$\mathrm{C}$ & 6.281080 & -2.444108 & 0.000000 \\
$\mathrm{C}$ & -6.281080 & 2.444108 & 0.000000 \\
$\mathrm{H}$ & 2.192050 & 2.149997 & 0.000000 \\
$\mathrm{H}$ & -2.192050 & -2.149997 & 0.000000 \\
$\mathrm{H}$ & 2.192050 & -2.149997 & 0.000000 \\
$\mathrm{H}$ & -2.192050 & 2.149997 & 0.000000 \\
$\mathrm{H}$ & 4.347102 & 3.394587 & 0.000000 \\
$\mathrm{H}$ & -4.347102 & -3.394587 & 0.000000 \\
$\mathrm{H}$ & 4.347102 & -3.394587 & 0.000000 \\
$\mathrm{H}$ & -4.347102 & 3.394587 & 0.000000 \\
$\mathrm{H}$ & 6.823561 & 3.385125 & 0.000000 \\
$\mathrm{H}$ & -6.823561 & -3.385125 & 0.000000 \\
$\mathrm{H}$ & 6.823561 & -3.385125 & 0.000000 \\
$\mathrm{H}$ & -6.823561 & 3.385125 & 0.000000 \\
$\mathrm{H}$ & 8.072388 & 1.245798 & 0.000000 \\
$\mathrm{H}$ & -8.072388 & -1.245798 & 0.000000 \\
$\mathrm{H}$ & 8.072388 & -1.245798 & 0.000000 \\
$\mathrm{H}$ & -8.072388 & 1.245798 & 0.000000 \\
\hline & & & \\
\hline & & & \\
\hline & & &
\end{tabular}


Table 6S: Cartesian coordinate of $\mathbf{6}$ (singlet) calculated at the UB3LYP/6-31G** level of approximation

\begin{tabular}{|c|c|c|c|}
\hline Atom type & $X$ & $\mathrm{Y}$ & $\mathrm{Z}$ \\
\hline $\mathrm{C}$ & 0.583913 & -0.337122 & 0.000000 \\
\hline $\mathrm{C}$ & -0.583913 & 0.337122 & 0.000000 \\
\hline $\mathrm{C}$ & 4.613729 & 1.216670 & 0.000000 \\
\hline $\mathrm{C}$ & -4.613729 & -1.216670 & 0.000000 \\
\hline $\mathrm{C}$ & 4.366115 & -0.190895 & 0.000000 \\
\hline $\mathrm{C}$ & -4.366115 & 0.190895 & 0.000000 \\
\hline $\mathrm{C}$ & 3.509098 & 2.126727 & 0.000000 \\
\hline $\mathrm{C}$ & -3.509098 & -2.126727 & 0.000000 \\
\hline $\mathrm{C}$ & 5.954825 & 1.711129 & 0.000000 \\
\hline $\mathrm{C}$ & -5.954825 & -1.711129 & 0.000000 \\
\hline $\mathrm{C}$ & 1.934974 & 0.231648 & 0.000000 \\
\hline $\mathrm{C}$ & -1.934974 & -0.231648 & 0.000000 \\
\hline $\mathrm{C}$ & 5.086658 & 3.995510 & 0.000000 \\
\hline $\mathrm{C}$ & -5.086658 & -3.995510 & 0.000000 \\
\hline $\mathrm{C}$ & 6.776637 & -0.590094 & 0.000000 \\
\hline $\mathrm{C}$ & -6.776637 & 0.590094 & 0.000000 \\
\hline $\mathrm{C}$ & 2.192312 & 1.614328 & 0.000000 \\
\hline $\mathrm{C}$ & -2.192312 & -1.614328 & 0.000000 \\
\hline $\mathrm{C}$ & 3.027269 & -0.648848 & 0.000000 \\
\hline $\mathrm{C}$ & -3.027269 & 0.648848 & 0.000000 \\
\hline $\mathrm{C}$ & 3.777827 & 3.521504 & 0.000000 \\
\hline $\mathrm{C}$ & -3.777827 & -3.521504 & 0.000000 \\
\hline $\mathrm{C}$ & 5.472708 & -1.078608 & 0.000000 \\
\hline $\mathrm{C}$ & -5.472708 & 1.078608 & 0.000000 \\
\hline $\mathrm{C}$ & 6.165352 & 3.114363 & 0.000000 \\
\hline $\mathrm{C}$ & -6.165352 & -3.114363 & 0.000000 \\
\hline
\end{tabular}




\begin{tabular}{|c|c|c|c|}
\hline $\mathrm{C}$ & 7.024882 & 0.780192 & 0.000000 \\
\hline $\mathrm{C}$ & -7.024882 & -0.780192 & 0.000000 \\
\hline $\mathrm{H}$ & 0.559567 & -1.425138 & 0.000000 \\
\hline $\mathrm{H}$ & -0.559567 & 1.425138 & 0.000000 \\
\hline $\mathrm{H}$ & 2.844578 & -1.720630 & 0.000000 \\
\hline $\mathrm{H}$ & -2.844578 & 1.720630 & 0.000000 \\
\hline $\mathrm{H}$ & 5.289108 & -2.149356 & 0.000000 \\
\hline $\mathrm{H}$ & -5.289108 & 2.149356 & 0.000000 \\
\hline $\mathrm{H}$ & 7.610215 & -1.286494 & 0.000000 \\
\hline $\mathrm{H}$ & -7.610215 & 1.286494 & 0.000000 \\
\hline $\mathrm{H}$ & 8.045744 & 1.151657 & 0.000000 \\
\hline $\mathrm{H}$ & -8.045744 & -1.151657 & 0.000000 \\
\hline $\mathrm{H}$ & 7.183107 & 3.494150 & 0.000000 \\
\hline $\mathrm{H}$ & -7.183107 & -3.494150 & 0.000000 \\
\hline $\mathrm{H}$ & 5.268796 & 5.066347 & 0.000000 \\
\hline $\mathrm{H}$ & -5.268796 & -5.066347 & 0.000000 \\
\hline $\mathrm{H}$ & 2.943815 & 4.217672 & 0.000000 \\
\hline $\mathrm{H}$ & -2.943815 & -4.217672 & 0.000000 \\
\hline $\mathrm{H}$ & 1.369708 & 2.322611 & 0.000000 \\
\hline $\mathrm{H}$ & -1.369708 & -2.322611 & 0.000000 \\
\hline
\end{tabular}


Table 7S: Cartesian coordinate of $\mathbf{1}$ (triplet) calculated at the UB3LYP/6-31G** level of approximation

\begin{tabular}{|c|c|c|c|}
\hline Atom type & $X$ & $\mathrm{Y}$ & $\mathrm{Z}$ \\
\hline $\mathrm{C}$ & 0.610403 & 0.000000 & 0.000000 \\
\hline $\mathrm{C}$ & -0.610403 & 0.000000 & 0.000000 \\
\hline $\mathrm{C}$ & 2.028607 & -0.001673 & 0.000000 \\
\hline $\mathrm{C}$ & -2.028607 & 0.001673 & 0.000000 \\
\hline $\mathrm{C}$ & 2.726625 & -1.225936 & 0.000000 \\
\hline $\mathrm{C}$ & -2.726625 & 1.225936 & 0.000000 \\
\hline $\mathrm{C}$ & 4.109575 & -1.269853 & 0.000000 \\
\hline $\mathrm{C}$ & -4.109575 & 1.269853 & 0.000000 \\
\hline $\mathrm{C}$ & 4.881082 & -0.078120 & 0.000000 \\
\hline $\mathrm{C}$ & -4.881082 & 0.078120 & 0.000000 \\
\hline $\mathrm{C}$ & 4.198852 & 1.179765 & 0.000000 \\
\hline $\mathrm{C}$ & -4.198852 & -1.179765 & 0.000000 \\
\hline $\mathrm{C}$ & 2.770008 & 1.231831 & 0.000000 \\
\hline $\mathrm{C}$ & -2.770008 & -1.231831 & 0.000000 \\
\hline $\mathrm{C}$ & 2.133146 & 2.495620 & 0.000000 \\
\hline $\mathrm{C}$ & -2.133146 & -2.495620 & 0.000000 \\
\hline $\mathrm{C}$ & 2.878760 & 3.672175 & 0.000000 \\
\hline $\mathrm{C}$ & -2.878760 & -3.672175 & 0.000000 \\
\hline $\mathrm{C}$ & 4.268647 & 3.634096 & 0.000000 \\
\hline $\mathrm{C}$ & -4.268647 & -3.634096 & 0.000000 \\
\hline $\mathrm{C}$ & 4.957004 & 2.395037 & 0.000000 \\
\hline $\mathrm{C}$ & -4.957004 & -2.395037 & 0.000000 \\
\hline $\mathrm{C}$ & 6.373612 & 2.320896 & 0.000000 \\
\hline $\mathrm{C}$ & -6.373612 & -2.320896 & 0.000000 \\
\hline $\mathrm{C}$ & 7.025030 & 1.091539 & 0.000000 \\
\hline $\mathrm{C}$ & -7.025030 & -1.091539 & 0.000000 \\
\hline
\end{tabular}




\begin{tabular}{lrrr}
$\mathrm{C}$ & 6.296748 & -0.095709 & 0.000000 \\
$\mathrm{C}$ & -6.296748 & 0.095709 & 0.000000 \\
$\mathrm{H}$ & 2.155271 & -2.148500 & 0.000000 \\
$\mathrm{H}$ & -2.155271 & 2.148500 & 0.000000 \\
$\mathrm{H}$ & 4.621607 & -2.227834 & 0.000000 \\
$\mathrm{H}$ & -4.621607 & 2.227834 & 0.000000 \\
$\mathrm{H}$ & 6.810001 & -1.053190 & 0.000000 \\
$\mathrm{H}$ & -6.810001 & 1.053190 & 0.000000 \\
$\mathrm{H}$ & 8.110583 & 1.056999 & 0.000000 \\
$\mathrm{H}$ & -8.110583 & -1.056999 & 0.000000 \\
$\mathrm{H}$ & 6.946276 & 3.244053 & 0.000000 \\
$\mathrm{H}$ & -6.946276 & -3.244053 & 0.000000 \\
$\mathrm{H}$ & 4.844068 & 4.555577 & 0.000000 \\
$\mathrm{H}$ & -4.844068 & -4.555577 & 0.000000 \\
$\mathrm{H}$ & 2.366157 & 4.629766 & 0.000000 \\
$\mathrm{H}$ & -2.366157 & -4.629766 & 0.000000 \\
$\mathrm{H}$ & 1.049416 & 2.532584 & 0.000000 \\
$\mathrm{H}$ & -1.049416 & -2.532584 & 0.000000 \\
\hline & & & \\
\hline & & & \\
\hline & & &
\end{tabular}


Table 8S: Cartesian coordinate of 2(triplet) calculated at the UB3LYP/6-31G** level of approximation

\begin{tabular}{cccl}
\hline Atom type & $\mathrm{X}$ & $\mathrm{Y}$ & $\mathrm{Z}$ \\
\hline $\mathrm{C}$ & 0.587942 & -0.339449 & 0.000000 \\
$\mathrm{C}$ & -0.587942 & 0.339449 & 0.000000 \\
$\mathrm{C}$ & 1.930294 & 0.232900 & 0.000000 \\
$\mathrm{C}$ & -1.930294 & -0.232900 & 0.000000 \\
$\mathrm{C}$ & 4.405083 & -0.017013 & 0.000000 \\
$\mathrm{C}$ & -4.405083 & 0.017013 & 0.000000 \\
$\mathrm{C}$ & 3.099465 & -0.616725 & 0.000000 \\
$\mathrm{C}$ & -3.099465 & 0.616725 & 0.000000 \\
$\mathrm{C}$ & 5.581488 & -0.836417 & 0.000000 \\
$\mathrm{C}$ & -5.581488 & 0.836417 & 0.000000 \\
$\mathrm{C}$ & 4.553723 & 1.405179 & 0.000000 \\
$\mathrm{C}$ & -4.553723 & -1.405179 & 0.000000 \\
$\mathrm{C}$ & 2.127517 & 1.629598 & 0.000000 \\
$\mathrm{C}$ & -2.127517 & -1.629598 & 0.000000 \\
$\mathrm{C}$ & 6.983015 & 1.167546 & 0.000000 \\
$\mathrm{C}$ & -6.983015 & -1.167546 & 0.000000 \\
$\mathrm{C}$ & 4.179562 & -2.823373 & 0.000000 \\
$\mathrm{C}$ & -4.179562 & 2.823373 & 0.000000 \\
$\mathrm{C}$ & 3.381829 & 2.204431 & 0.000000 \\
$\mathrm{C}$ & -3.381829 & -2.204431 & 0.000000 \\
$\mathrm{C}$ & 6.857938 & -0.217123 & 0.000000 \\
$\mathrm{C}$ & -6.857938 & 0.217123 & 0.000000 \\
$\mathrm{C}$ & 3.031337 & -2.035070 & 0.000000 \\
$\mathrm{C}$ & -3.031337 & 2.035070 & 0.000000 \\
$\mathrm{C}$ & 5.848706 & 1.974908 & 0.000000 \\
$\mathrm{C}$ & -1.974908 & 0.000000 \\
$\mathrm{C}$ & -2.245724 & 0.000000
\end{tabular}




\begin{tabular}{lrrr}
$\mathrm{C}$ & -5.441867 & 2.245724 & 0.000000 \\
$\mathrm{H}$ & 0.539622 & -1.421084 & 0.000000 \\
$\mathrm{H}$ & -0.539622 & 1.421084 & 0.000000 \\
$\mathrm{H}$ & 1.263733 & 2.285307 & 0.000000 \\
$\mathrm{H}$ & -1.263733 & -2.285307 & 0.000000 \\
$\mathrm{H}$ & 7.969712 & 1.621413 & 0.000000 \\
$\mathrm{H}$ & -7.969712 & -1.621413 & 0.000000 \\
$\mathrm{H}$ & 4.080502 & -3.905034 & 0.000000 \\
$\mathrm{H}$ & -4.080502 & 3.905034 & 0.000000 \\
$\mathrm{H}$ & 3.483626 & 3.286117 & 0.000000 \\
$\mathrm{H}$ & -3.483626 & -3.286117 & 0.000000 \\
$\mathrm{H}$ & 5.944896 & 3.057051 & 0.000000 \\
$\mathrm{H}$ & -5.944896 & -3.057051 & 0.000000 \\
$\mathrm{H}$ & 7.742888 & -0.847281 & 0.000000 \\
$\mathrm{H}$ & -7.742888 & 0.847281 & 0.000000 \\
$\mathrm{H}$ & 6.333842 & -2.865665 & 0.000000 \\
$\mathrm{H}$ & -6.333842 & 2.865665 & 0.000000 \\
$\mathrm{H}$ & 2.071272 & -2.535421 & 0.000000 \\
$\mathrm{H}$ & -2.071272 & 2.535421 & 0.000000 \\
\hline & & & \\
\hline & & &
\end{tabular}


Table 9S: Cartesian coordinate of 5(triplet) calculated at the UB3LYP/6-31G** level of approximation

\begin{tabular}{llll}
\hline Atom type & $\mathrm{X}$ & $\mathrm{Y}$ & $\mathrm{Z}$ \\
\hline $\mathrm{C}$ & 0.607893 & 0.000000 & 0.000000 \\
$\mathrm{C}$ & -0.607893 & 0.000000 & 0.000000 \\
$\mathrm{C}$ & 2.034776 & 0.000000 & 0.000000 \\
$\mathrm{C}$ & -2.034776 & 0.000000 & 0.000000 \\
$\mathrm{C}$ & 4.872904 & 0.000000 & 0.000000 \\
$\mathrm{C}$ & -4.872904 & 0.000000 & 0.000000 \\
$\mathrm{C}$ & 4.155525 & 1.238012 & 0.000000 \\
$\mathrm{C}$ & -4.155525 & -1.238012 & 0.000000 \\
$\mathrm{C}$ & 4.155525 & -1.238012 & 0.000000 \\
$\mathrm{C}$ & -4.155525 & 1.238012 & 0.000000 \\
$\mathrm{C}$ & 6.303053 & 0.000000 & 0.000000 \\
$\mathrm{C}$ & -6.303053 & 0.000000 & 0.000000 \\
$\mathrm{C}$ & 2.742215 & 1.213915 & 0.000000 \\
$\mathrm{C}$ & -2.742215 & -1.213915 & 0.000000 \\
$\mathrm{C}$ & 2.742215 & -1.213915 & 0.000000 \\
$\mathrm{C}$ & -2.742215 & 1.213915 & 0.000000 \\
$\mathrm{C}$ & 4.889540 & 2.453474 & 0.000000 \\
$\mathrm{C}$ & -4.889540 & -2.453474 & 0.000000 \\
$\mathrm{C}$ & 4.889540 & -2.453474 & 0.000000 \\
$\mathrm{C}$ & -4.889540 & 2.453474 & 0.000000 \\
$\mathrm{C}$ & 6.986313 & 1.243058 & 0.000000 \\
$\mathrm{C}$ & -6.986313 & -1.243058 & 0.000000 \\
$\mathrm{C}$ & 6.986313 & -1.243058 & 0.000000 \\
$\mathrm{C}$ & -6.986313 & 1.243058 & 0.000000 \\
$\mathrm{C}$ & 6.281150 & 2.444225 & 0.000000 \\
$\mathrm{C}$ & -6.281150 & -2.444225 & 0.000000
\end{tabular}




\begin{tabular}{lrrr}
$\mathrm{C}$ & 6.281150 & -2.444225 & 0.000000 \\
$\mathrm{C}$ & -6.281150 & 2.444225 & 0.000000 \\
$\mathrm{H}$ & 2.192340 & 2.149560 & 0.000000 \\
$\mathrm{H}$ & -2.192340 & -2.149560 & 0.000000 \\
$\mathrm{H}$ & 2.192340 & -2.149560 & 0.000000 \\
$\mathrm{H}$ & -2.192340 & 2.149560 & 0.000000 \\
$\mathrm{H}$ & 4.347356 & 3.394678 & 0.000000 \\
$\mathrm{H}$ & -4.347356 & -3.394678 & 0.000000 \\
$\mathrm{H}$ & 4.347356 & -3.394678 & 0.000000 \\
$\mathrm{H}$ & -4.347356 & 3.394678 & 0.000000 \\
$\mathrm{H}$ & 6.823803 & 3.385105 & 0.000000 \\
$\mathrm{H}$ & -6.823803 & -3.385105 & 0.000000 \\
$\mathrm{H}$ & 6.823803 & -3.385105 & 0.000000 \\
$\mathrm{H}$ & -6.823803 & 3.385105 & 0.000000 \\
$\mathrm{H}$ & 8.072626 & 1.245930 & 0.000000 \\
$\mathrm{H}$ & -8.072626 & -1.245930 & 0.000000 \\
$\mathrm{H}$ & 8.072626 & -1.245930 & 0.000000 \\
$\mathrm{H}$ & -8.072626 & 1.245930 & 0.000000 \\
\hline & & & \\
\hline & & & \\
\hline & & &
\end{tabular}


Table 10S: Cartesian coordinate of 6 (triplet) calculated at the UB3LYP/6-31G** level of approximation

\begin{tabular}{|c|c|c|c|}
\hline Atom type & $X$ & $\mathrm{Y}$ & $\mathrm{Z}$ \\
\hline $\mathrm{C}$ & 0.583305 & -0.336771 & 0.000000 \\
\hline $\mathrm{C}$ & -0.583305 & 0.336771 & 0.000000 \\
\hline $\mathrm{C}$ & 4.612664 & 1.218961 & 0.000000 \\
\hline $\mathrm{C}$ & -4.612664 & -1.218961 & 0.000000 \\
\hline $\mathrm{C}$ & 4.366104 & -0.188478 & 0.000000 \\
\hline $\mathrm{C}$ & -4.366104 & 0.188478 & 0.000000 \\
\hline $\mathrm{C}$ & 3.508035 & 2.128667 & 0.000000 \\
\hline $\mathrm{C}$ & -3.508035 & -2.128667 & 0.000000 \\
\hline $\mathrm{C}$ & 5.953881 & 1.714091 & 0.000000 \\
\hline $\mathrm{C}$ & -5.953881 & -1.714091 & 0.000000 \\
\hline $\mathrm{C}$ & 1.935917 & 0.233181 & 0.000000 \\
\hline $\mathrm{C}$ & -1.935917 & -0.233181 & 0.000000 \\
\hline $\mathrm{C}$ & 5.084822 & 3.998246 & 0.000000 \\
\hline $\mathrm{C}$ & -5.084822 & -3.998246 & 0.000000 \\
\hline $\mathrm{C}$ & 6.776771 & -0.586886 & 0.000000 \\
\hline $\mathrm{C}$ & -6.776771 & 0.586886 & 0.000000 \\
\hline $\mathrm{C}$ & 2.191939 & 1.615504 & 0.000000 \\
\hline $\mathrm{C}$ & -2.191939 & -1.615504 & 0.000000 \\
\hline $\mathrm{C}$ & 3.027531 & -0.646823 & 0.000000 \\
\hline $\mathrm{C}$ & -3.027531 & 0.646823 & 0.000000 \\
\hline $\mathrm{C}$ & 3.776418 & 3.523942 & 0.000000 \\
\hline $\mathrm{C}$ & -3.776418 & -3.523942 & 0.000000 \\
\hline $\mathrm{C}$ & 5.473354 & -1.075913 & 0.000000 \\
\hline $\mathrm{C}$ & -5.473354 & 1.075913 & 0.000000 \\
\hline $\mathrm{C}$ & 6.163950 & 3.117253 & 0.000000 \\
\hline $\mathrm{C}$ & -6.163950 & -3.117253 & 0.000000 \\
\hline
\end{tabular}




\begin{tabular}{|c|c|c|c|}
\hline $\mathrm{C}$ & 7.024226 & 0.783793 & 0.000000 \\
\hline $\mathrm{C}$ & -7.024226 & -0.783793 & 0.000000 \\
\hline $\mathrm{H}$ & 0.559715 & -1.424806 & 0.000000 \\
\hline $\mathrm{H}$ & -0.559715 & 1.424806 & 0.000000 \\
\hline $\mathrm{H}$ & 2.845021 & -1.718661 & 0.000000 \\
\hline $\mathrm{H}$ & -2.845021 & 1.718661 & 0.000000 \\
\hline $\mathrm{H}$ & 5.290064 & -2.146723 & 0.000000 \\
\hline $\mathrm{H}$ & -5.290064 & 2.146723 & 0.000000 \\
\hline $\mathrm{H}$ & 7.610798 & -1.282756 & 0.000000 \\
\hline $\mathrm{H}$ & -7.610798 & 1.282756 & 0.000000 \\
\hline $\mathrm{H}$ & 8.044930 & 1.155730 & 0.000000 \\
\hline $\mathrm{H}$ & -8.044930 & -1.155730 & 0.000000 \\
\hline $\mathrm{H}$ & 7.181593 & 3.497363 & 0.000000 \\
\hline $\mathrm{H}$ & -7.181593 & -3.497363 & 0.000000 \\
\hline $\mathrm{H}$ & 5.266706 & 5.069137 & 0.000000 \\
\hline $\mathrm{H}$ & -5.266706 & -5.069137 & 0.000000 \\
\hline $\mathrm{H}$ & 2.942048 & 4.219686 & 0.000000 \\
\hline $\mathrm{H}$ & -2.942048 & -4.219686 & 0.000000 \\
\hline $\mathrm{H}$ & 1.368718 & 2.323094 & 0.000000 \\
\hline $\mathrm{H}$ & -1.368718 & -2.323094 & 0.000000 \\
\hline
\end{tabular}


Table 11S: Spin expectation values $\left\langle S^{2}>\right.$ of 1(singlet), 2(singlet), 5(singlet), 6(singlet), 1(triplet), 2(triplet), 5(triplet) and 6(triplet) evaluated at the UBHandHLYP/6-31G* level of approximation

\begin{tabular}{cc}
\hline System & $<S^{2}>$ \\
\hline $\mathbf{1}$ (singlet) & 1.2523 \\
$\mathbf{2}$ (singlet) & 1.0581 \\
$\mathbf{5}$ (singlet) & 1.5179 \\
$\mathbf{6}$ (singlet) & 1.5744 \\
$\mathbf{1}$ (triplet) & 2.3729 \\
$\mathbf{2}$ (triplet) & 2.3696 \\
$\mathbf{5}$ (triplet) & 2.4180 \\
$\mathbf{6}$ (triplet) & 2.4157 \\
\hline
\end{tabular}

